\title{
Descubrimiento de la testosterona
}

\section{Discovery of testosterone}

\author{
Jácome $A^{1}$.
}

${ }^{1}$ Internista-Endocrinólogo. Expresidente y Miembro Honorario, Asociación Colombiana de Endocrinología, Diabetes y Metabolismo. Miembro de Número, Academia Nacional de Medicina.

Fecha de recepción: 27/05/2019

Fecha de aceptación: 31/07/2019

\section{Resumen}

Los efectos de la castración en animales y en hombres eunucos eran conocidos desde la antigüedad. Tomó siglos comprender que había una sustancia cuya disminución o franca ausencia también generaba estos efectos en la vejez. El hombre empezó a buscar la fuente de la juventud y del desempeño viril. ¿Acaso la encontró Ponce de León en aguas de San Agustín, en la Florida? Ciertamente no. Algo más racional fue el uso de la organoterapia testicular como terapia de reemplazo (o suplencia). A comienzos del siglo XX estuvieron de moda los trasplantes testiculares para lograr un rejuvenecimiento, pero solo tenían un corto efecto placebo.

Esta revisión narrativa tiene por objeto describir los hechos que llevaron al descubrimiento de los esteroides y en particular de la testosterona. Esto se logró en la "la edad heroica de la endocrinología reproductiva", así llamada porque se usaban toneladas de órganos animales o miles de litros de orina para obtener unos pocos miligramos de hormona. Los compuestos con un anillo esteroideo se categorizaron en andrógenos, estrógenos, progestágenos, glucocorticoides y mineralocorticoides, según su efecto.

En 1935, Ernst Laqueur (Ámsterdam) aisló la testosterona y Adolf Butenandt (Gdansk) y Leopold Ruzicka (Zürich) la sintetizaron luego. Esto se logró en los albores de la Segunda Guerra Mundial, gracias al apoyo de las farmacéuticas. En este trabajo participaron científicos nazis que posteriormente pudieron seguir trabajando en sus laboratorios.

¿Tendría la hormona de la virilidad efecto positivo en la combatividad de los soldados? Los nazis y también los soviéticos así lo creyeron. Estos últimos extendieron su uso a los deportistas, en su afán de propaganda.

La dificultad de conseguir niveles hormonales estables ha dirigido la investigación hacia la administración de diversas sales por diferentes vías. Ampliar las indicaciones médicas de la testosterona más allá del tratamiento del hipogonadismo masculino tampoco ha sido fácil. Sus metabolitos, los esteroides anabólicos, estuvieron de moda por unas décadas, pero actualmente se han convertido en sustancias prohibidas por el dopaje de atletas, excepto por su uso en pacientes con sida. No hay un acuerdo sobre su administración y riesgos de uso en los adultos mayores, aunque parece que su formulación no aumenta la posibilidad de desarrollar cáncer de próstata. Desde luego, las preparaciones de testosterona han venido mejorándose en los últimos años para lograr niveles fisiológicos y permanentes en los hipogonádicos.

Palabras clave: testosterona, andrógenos, hipogonadismo.

\section{Abstract}

The effects of castration in animals and human eunuchs were known since antiquity. It took milleniae to understand there was a substance whose decrease or frank absence also generated such ageing effects. Man started to look for the fountain of youth and viril performance. Was it found by spaniard conqueror Ponce de Leon in waters of St.Augustine, Florida? Certainly not. More rational were testicular organotherapy as "replacement therapy" and a century ago, testicular "rejuvenation" transplants. Those therapies were fashionable although innocuous (short placebo effect), and rather a matter of quackery. Discovery of steroids in the so-called "heroic age of reproductive endocrinology" when tons of animal organs and thousands of liters of urine were used only to obtain a few miligramas of active substance, androgens, estrogens, progestagens, glucocorticoids and mineralocorticoids were found.

This narrative review paper aims to describe historical facts that led to discovery of steroids, specially testosterone. These were achieved during the "golden age of reproductive endocrinology", so called because tons of animal organs or thousands of urine liters were used in order to obtain a few milligrams of hormone; compouds with the steroid ring were classified as androgens, estrogens, progestagens, glucocorticoids and mineralocorticoids, according to their tissue effects.

Ernst Laqueur (Amsterdam) isolated testosterone in 1935, Adolf Butenandt (Gdansk) and Leopold Ruzicka (Zürich) synthetized it shortly therefore. This was done in the beginning of 
World War II, thanks to big pharma support; in the work, Nazi scientists participated who later on were allowed to continue their work. Would the virility hormone have a positive effect on soldiers combativity? Nazis and Soviets so believed. The latter also gave it to their athletes, looking for a "propaganda" effect.

Difficulty in obtaining stable hormonal levels led researchers for testosterone salts to be administered by different routes. But besides true cases of male hypogonadism other indications were not clear. Anabolic steroids are metabolites that were fashionable for a couple of decades although -except for AIDS cases-are no longer prescribed. They are scheduled substances for athletes. No agreement has been reached in regard to some indications in senior adults although its seems to be that testosterone administration does not increase prostate cancer risk. Therapeutic agents have been improved in recent years to obtain physiologic and permanent levels in hypogonadic patients.

Keywords: Testosterone, androgens, hypogonadism.

\section{Introducción}

Desde la antigüedad se conocía que la virilidad tenía una relación con la gónada masculina, que era el testigo o testículo del varón. Después de siglos de gallos capones, de toros castrados o bueyes y de la presencia de eunucos en las diferentes culturas, el ser humano sabía que la fuerza física, la potencia sexual, la apariencia masculina y la capacidad para la lucha eran propias del hombre, particularmente del joven. Con el pasar de los años, algunas de estas características iban desvaneciéndose, por lo que se consideraba que en dicha gónada residía la fuente de la juventud, que podía recuperarse con organoterapia o con trasplantes. Empezó a discutirse el tema de un posible climaterio masculino ${ }^{(1)}$.

Los griegos habían hecho elucubraciones sobre la virilidad, la vejez y la fertilidad, pero solo hasta 1849, Berthold, a la sazón de director de un zoológico, hizo un experimento sencillo, aunque paradigmático: tomó 6 gallos, a un par los dejo como testigos sanos y a cuatro los castró, pero solo a dos los trasplantó con sus testículos cambiados a la cavidad abdominal. Estos cacareaban mucho, peleaban entre sí y con otros gallos jóvenes y mostraban inclinación normal hacia las gallinas. Como ya estos testículos no estaban en conexión con los nervios originales debían, entonces, actuar a través de la sangre. Por envidia de un colega con un cargo administrativamente superior que criticó la investigación, el trabajo cayó en el olvido por varias décadas ${ }^{(2)}$.

Tomó décadas desempolvar estos datos correctos, pero se produjo una revolución mediática cuando el famoso investigador Brown-Sequard afirmó 4 décadas después haberse rejuvenecido 20 años al aplicarse extracto testicular acuoso. Esta falseada conclusión se publicó nada menos que en el Lancet de Londres. En medio de la seudociencia y la charlatanería, científicos disciplinados empezaron a aportar datos. En la década de 1920, Nussbaum y Steinach lograron resultados similares de los de Berthold: el primero en ranas y el segundo en ratas. Pezard, bajo la tutela del fisiólogo Gley y trabajando estrechamente con Caridroit y Sand, confirmó los hallazgos de Berthold también en gallos y llamó a la supuesta sustancia que pasaba a la sangre en el gallo trasplantado la hormona testicular ${ }^{(3)}$. En 1929, Funk y Harrow, con base en los resultados del trabajo de los franceses, pensaron con lógica que si la hormona testicular se transportaba por la sangre para ir a los órganos sexuales secundarios, dicha hormona debería filtrarse por el riñón y aparecer en la orina ${ }^{(2)}$.

\section{La edad de oro de la química de los esteroides}

La bioquímica solidificó la endocrinología, pues podía identificar moléculas. Al tratar la orina concentrada con alcohol, acidificándola, extrayendo cloroformo e hidrolizándola, en los gallos castrados inyectados con la sustancia presente en dicha orina crecían las crestas. Los bioquímicos estaban conscientes de las bajas concentraciones tisulares de las moléculas, por lo que se requerirían grandes cantidades de órganos de mamíferos $^{(4)}$.

Para 1920, el profesor de Química Fisiológica de la Universidad de Chicago, Fred Conrad Koch, en cuya memoria se otorga actualmente un prestigioso premio de endocrinología, empezó a colar testículos de toro para obtener extractos que inyectaba a gallos capones para ver el desarrollo de la cresta, cosa que solo logró cuando se le unió Gallagher, pues con unos procesos de extracción que involucraban varias etapas lograron una mezcla activa, ya que la cresta del capón respondía en 5 días a la administración de solo 0,01 mg de la nueva sustancia.

En 1927, Koch logró tener fácil acceso a una gran fuente de testículos bovinos en la Union Stockyard \& Transit de Chicago, una enorme carnicería y centro de empaquetamiento que perteneció a los ferrocarriles, con Vanderbilt como inversionista. Koch logró convencer a sus estudiantes para que le ayudaran en el tedioso trabajo de extraer las hormonas. Ese mismo año, Koch y su estudiante McGee lograron obtener el primer extracto activo con $20 \mathrm{mg}$ de una sustancia (extraída de 40 libras de la fracción lipídica de testículos de toro), que al ser inyectadas en gallos, ratas y cerdos castrados les hacían recuperar sus rasgos de machos $^{(2)}$.

En la década de 1920 se habían conformado grupos en Estados Unidos y en Europa que trabajaban en la búsqueda de los esteroides sexuales. Los estudios realizados en la Universidad Washington en San Luis por Allen y Doisy y los estudios concomitantes de alemanes y holandeses, con el apoyo de la industria farmacéutica, fueron fundamentales para el descubrimiento de los diversos estrógenos, progestágenos y andrógenos ${ }^{(5-9)}$.

Un paso gigante se dio cuando se descubrió la estructura de los anillos esteroidales; por un lado, estuvo el alemán Wieland 
(quien desde la Academia Bávara de Ciencias en Múnich estudió los ácidos y sales biliares) y por otro Windaus en Gottingen (que siguió los pasos de la transformación del colesterol en vitamina $\mathrm{D}_{3}$ o colecalciferol) al identificar la estructura fenantrénica de estos esteroides. Estos alemanes precedieron en el Nobel de Química a Butenandt, quien fue discípulo de Windaus ${ }^{(2)}$.

Para 1929, Doisy, y 2 meses más tarde Butenandt, comunicaron, cada cual por su lado, el aislamiento de la estrona de la orina de mujeres embarazadas y al año siguiente se descubrió la estructura de la estrona cristalizada. El descubrimiento de la estrona fue muy importante, ya que los químicos aprendieron a manipular las moléculas esteroides, lo que genera la posibilidad de su síntesis química. La orina de yeguas preñadas comenzó a ser la fuente de estrógenos conjugados. Paralelamente, Moore (del grupo de Laqueur) estandarizó la prueba de la cresta del gallo capón para medir la actividad androgénica en tejidos y líquidos orgánicos, así como también en soluciones químicas ${ }^{(7)}$. En 1931, Butenandt aisló la androsterona (tabla 1) como una sustancia químicamente pura ${ }^{(10,11)}$. El nombre vino por el núcleo policíclico parecido al del colesterol (en aquellas épocas se llamaba colesterina), con un grupo hidroxilo y otro de cetona (andro, esterol, cetona, o androsterona) ${ }^{(11)}$.

Las dificultades con la purificación, análisis estructural y síntesis de eventuales hormonas presentes en sangre, orina o tejidos lo comentan la historiadora Wilson de esta forma ${ }^{(8)}$ : "Durante las etapas de purificación, los bioensayos disponibles (ineficientes y complicados), consumían buena parte de los extractos; las técnicas de purificación eran primitivas y además los extractos solo contenían trazas de las hormonas. Para los análisis estructurales, los métodos espectroscópicos eran inadecuados. Para 1926 solo 3 hormonas se había cristalizado: la epinefrina, la tiroxina y la insulina" (tabla 1).
Tiempo después, el Nobel Schally requeriría medio millón de hipotálamos porcinos para aislar sus hormonas liberadoras. No es de sorprender que la casa farmacéutica Armour, especializada en la fabricación de hormonas (tiroides desecada, ACTH, TSH, PTH), fuera filial de la compañía de carnes Armour, que incursionó antes en la organoterapia. Como quien dice era un negocio de carniceros ${ }^{(1)}$.

Varias casas farmacéuticas europeas: Schering de Berlín, Organon de Holanda y Ciba de Suiza, estaban ya invirtiendo dinero suficiente en investigación sobre esteroides y se asociaban con los centros académicos ${ }^{(2)}$. También estuvieron involucradas en estudios y patentes de esteroles las empresas alemanas Merck y Bayer (a través de Windaus) y Boehringer Ingelheim (a través de Wieland).

Al mejorar la técnica de Koch, Laqueur, fundador de Organon en Ámsterdam, logró aislar la hormona androgénica que llamó testosterona en 1934. En esta empresa trabajó en el aislamiento de estrógenos y testosterona y fue el primero en producir insulina para el continente europeo. Butenandt logró identificar la estructura mientras trabajaba para la Schering alemana en el Instituto Químico de la Universidad Técnica en Gdansk. Independientemente, y casi al mismo tiempo, Ruzicka, con el grupo Ciba en Zúrich, logró la síntesis parcial de la molécula de testosterona, de cuya estructura básica se podían obtener cientos de compuestos cuando se aplicaban métodos químicos de oxidación y reducción ${ }^{(12)}$.

\section{Guerras y ciencia}

Los proyectos científicos se desarrollan en épocas de guerra y paz, y los investigadores se relacionan de alguna manera con una u otra ideología. El caso de la investigación en esteroides sexuales no es la excepción. Laqueur, por ejemplo, era

Tabla 1. La edad heroica del descubrimiento de las hormonas esteroideas: de 1929 a 1954 . Con datos de Fieser \& Fieser y de Tausk ${ }^{(8)}$

\begin{tabular}{l|l|l} 
& \multicolumn{1}{c|}{ Hormona } & \multicolumn{1}{c}{ Material necesario } \\
\hline Estrona & Cientos de litros de orina femenina & Schering \\
\hline Estradiol & 4 toneladas de ovarios de cerdo & \\
\hline Progesterona & $20 \mathrm{~kg}$ de cuerpos lúteos de 50000 cerdos & Schering y Squibb \\
\hline Vitamina $\mathrm{D}_{2}$ & $8 \mathrm{~g}$ de ergosterol irradiado & IG Farben/Bayer \\
\hline Androsterona* & $15000 \mathrm{~L}$ de orina de humana & Schering \\
\hline Testosterona & Toneladas de testículos de toro & Organón \\
\hline Corticosterona/DOCA & Suprarrenales bovinas (1 ton) & Ciba y Organón \\
\hline Cortisona & Suprarrenales bovinas (150 ton) $)^{* *}$ & Parke Davis, Merck \\
\hline Aldosterona & Suprarrenales bovinas ( 12 ton) & Ciba \\
\hline
\end{tabular}

*Butenandt había aislado unos 15 mg de androsterona (la primera hormona esteroide descubierta) con toda esta cantidad de orina. Ruzicka sintetizó la androsterona como la primera hormona esteroidea.

**Esta es la cantidad que solo el laboratorio de Kendall procesó en un período de 20 años. Tiempo más tarde Schally necesitaría medio millón de hipotálamos de cerdo para identificar la TRH y luego sintetizarla. 
un humanista que insistió que la compañía Organon donara un $10 \%$ de sus utilidades para uso en investigación. Estudió la forma de ayudar a las víctimas del gas mostaza de la Primera Guerra y en la Segunda, ayudó a los refugiados perseguidos por los nazis en Holanda. Estos finalmente entraron a su país y perdió sus posiciones académicas, pero logró evitar que le pusieran en su ropa la estrella de David amarilla que identificada a los judíos. Sin embargo, en 1943 fue deportado a un campo de concentración.

Windaus no simpatizaba con el régimen nazi, pero pudo continuar sus investigaciones durante el período del Nacional Socialismo por la reputación que tenía. Como científico de Schering alemana, Windaus le sugirió a Butenandt que estudiara las hormonas ováricas. Aunque nunca se supo claramente cuál fue su orientación política, él firmó un documento con otros profesores alemanes jurando lealtad a Hitler y a su estado nacional socialista. Se afilió a este partido (con el numero 3716562) y como director de un instituto científico de importancia solicitó fondos para investigar en proyectos militares de utilidad para la guerra.

El gobierno nazi obligó a Butenandt y a Ruzicka a rechazar el Premio Nobel que habían ganado por sus estudios sobre la testosterona, pues se consideraba que otros pueblos no tenían derecho a juzgar el éxito de científicos del Tercer Reich. Ambos continuaron sus investigaciones durante y después de la guerra y en 1948 viajaron a recibir el Premio Nobel $^{(2)}$.

Se dice que los soldados nazis recibían testosterona para aumentar su agresividad y que en los campos de concentración se experimentaba con esta hormona en humanos. Parece también cierto que a Hitler le aplicaban inyecciones de testosterona. Algunas farmacéuticas alemanas se vieron involucradas con el régimen, pero también los rusos usaron luego sustancias androgénicas, en particular anabólicos para sus $\operatorname{atletas}^{(13-15)}$.

\section{Usos y abusos de las hormonas masculinas}

En un principio no se entendía bien cuál era la función concreta de la testosterona. Se sabía su influencia en la fuerza y posible rejuvenecimiento, pero se ignoraba la función de la hormona en el crecimiento muscular a través de la síntesis de las proteínas; es decir, que era una hormona anabólica y aumentaba la retención nitrogenada. Rápidamente se obtuvieron esteres de testosterona para su uso por vía oral, inyectable, en forma de pellets y por parches transdérmicos. Desde entonces se han usado estos diferentes compuestos en diferentes indicaciones aprobadas y no aprobadas, como en el hipogo- nadismo masculino, en la deficiencia hormonal asociada con el envejecimiento, en la disfunción eréctil, como afrodisiaco, como estimulante de la producción de hematíes, entre otras. Sus derivados, llamados anabólicos, se usaron en el crecimiento de niños con baja estatura, en los pacientes desnutridos con sida y como sistema prohibido de dopaje en los atletas ${ }^{(3)}$.

Como su administración fortalecía los músculos, esto dio origen al uso ulterior de anabólicos por atletas y hombres interesados en desarrollar una espectacular musculatura, pues se mejoraban los resultados de extenuantes ejercicios gimnásticos. El uso indiscriminado e ilegal de estos anabólicos en los gimnastas que competían en juegos olímpicos tuvo su punto culminante en la pérdida de la medalla otorgada al corredor Ben Johnson en Seúl en 1988. El problema de dopaje de deportistas en el mundo es inacabable con estas hormonas, la eritropoyetina y otras sustancias de una larga lista ${ }^{(1)}$.

La suplencia androgénica no se ha usado tan frecuentemente como la estrogénica. Por supuesto que desde finales de 1800 se han usado estos preparados con fines de rejuvenecimiento, recuperación de la potencia, tratamiento de la homosexualidad masculina, aumento de la libido, prevención de la osteoporosis o utilización de sus propiedades anabólicas. Los parches de testosterona disponibles, o bien resultan inconvenientes por su necesidad de aplicarse diariamente sobre el escroto o producen dermatitis de contacto.

La testosterona más barata y más usada en hipogonadismo masculino es el dipropionato para aplicación parenteral de depósito, pero tiene el problema de los picos altos iniciales y los niveles bajos posteriores, lo que además genera preocupación sobre sus efectos en la próstata, pues desde la década de 1940 se empezó a hablar de la influencia de los niveles de testosterona plasmática en la generación de cáncer de próstata. Esta teoría ha perdido vigencia últimamente, pues la mayoría de los pacientes con cáncer de próstata tienen niveles de testosterona considerados normales y al parecer lo que importa es la concentración de dihidrotestosterona prostática, que viene de la testosterona por acción de la 5-alfa-reductasa. El conocimiento de esta enzima llevó al uso de sus inhibidores y de otros antiandrógenos para el tratamiento de enfermedades prostáticas y de estados hiperandrogénicos en la mujer ${ }^{(16-24)}$.

Recientemente se ha publicado una revisión muy completa del tema de este artículo, la cual lleva la firma de Eberhard y Susan Nieschlag, dos importantes investigadores en este campo ${ }^{(5)}$. Los colombianos tenemos en el urólogo javeriano y profesor de la Universidad de Queens en Kingston, Ontario, Álvaro Morales Gómez, un investigador de talla mundial en este tema de la testosterona y del declive hormonal del envejecimiento masculino ${ }^{(9,20)}$. 


\section{Referencias}

1. Jácome A. Historia de las hormonas. Academia Nacional de Medicina. 2008.

2. Medvei VC. A history of endocrinology. Minghan, MA: MTP Press Ltd. 1982

3. Pezard A. Complete dictionary of scientific biography. 2008 Disponible en: https://www.encyclopedia.com/science/dictionaries-thesaurusespictures-and-press-releases/pezard-albert

4. Simpson E. Clinical biochemistry in Scotland, a random history. 2018. Disponible en: http://www.elliottsimpson.com/history/introduction.html

5. Nieschlag E, Nieschlag S. The history of discovery, synthesis and development of testosterone for clinical use. Eur J Endocrinol. 2019;180(6):R201-12.

6. Freeman ER, Bloom DA, McGuire EJ. A brief history of testosterone. J Urol. 2001;165(2):371-3

7. Parkes AS. The rise of reproductive endocrinology 1926-40. Proc Sec Endocr. 1966;34:19-32.

8. Wilson JD. The evolution of endocrinology. Clin Endocrinol. 2005;62(4):389-96

9. Morales A. The long and tortuous history of the discovery of testosterone and its clinical application. J Sex Med. 2013;10(4):1178-83.

10. Nieschlag E, Nieschlag S. Testosterone deficiency: a historical perspective. Asian J Androl. 2014;16(2):161-8.

11. Gaudillière JP. Better prepared than synthesized: Adolf Butenandt, Schering $\mathrm{Ag}$ and the transformation of sex steroids into drugs (1930-1946). Stud Hist Philos Biol Biomed Sci. 2005;36(4):612-44.

12. Morgentaler A, Traish A. The history of testosterone and the evolution of its therapeutic potential. Nat Clin Urol. 2006;3(5):260-7.

13. Dotson JL, Brown RT. The history of the development of anabolic-androgenic steroids. Pediatr Clin North Am. 2007;54(4):761-9.
14. Associated Press. Soviet doping widespread, report says: magazine claims athletes had secret lab near Seoul. 1989. Disponible en: https://www.latimes.com/archives/la-xpm-1989-03-24-sp-460-story.html

15. Peters J. The man behind the juice: fifty years ago, a doctor brought steroids to America. 2005. Disponible en: https://slate.com/culture/2005/02/ the-doctor-who-brought-steroids-to-america.html

16. Gan EH, Pattman S, Pearce S, Quinton R. Many men are receiving unnecessary testosterone prescriptions. BMJ. 2012;345:e5469.

17. Cunningham GR. Testosterone replacement therapy for late-onset hypogonadism. Nat Clin Pract Urol. 2006;3(5):260-7.

18. Sansone A, Sansone M, Lenzi A, Romanelli F. Testosterone replacement therapy: The Emperor's New Clothes. Rejuvenation Res. 2017;20(1):9-14.

19. Klinge CM, Clark BJ, Prough RA. Dehydroepiandrosterone research: past, current, and future. Vitam Horm. 2018;108:1-28.

20. Morales A. Andropausia. 2000. Disponible en: https://encolombia.com/ medicina/revistas-medicas/academedicina/va-56/medicina23201-andropausia/

21. Hoberman JM, Yesalis CE. The history of synthetic testosterone. Sci Am 1995;272(2):76-81.

22. Matsumoto AM. Testosterone administration in older men. Postgrad Med 2008;120(3):130-53.

23. Miner M, Canty DJ, Shabsigh R. Testosterone replacement therapy in hypogonadal men: assessing benefits, risks, and best practices. Postgrad Med. 2008;120(4):114.

24. Wilson JD, Griffin JE. The use and misuse of androgens. Metabolism 1980;29(12):1278-95.
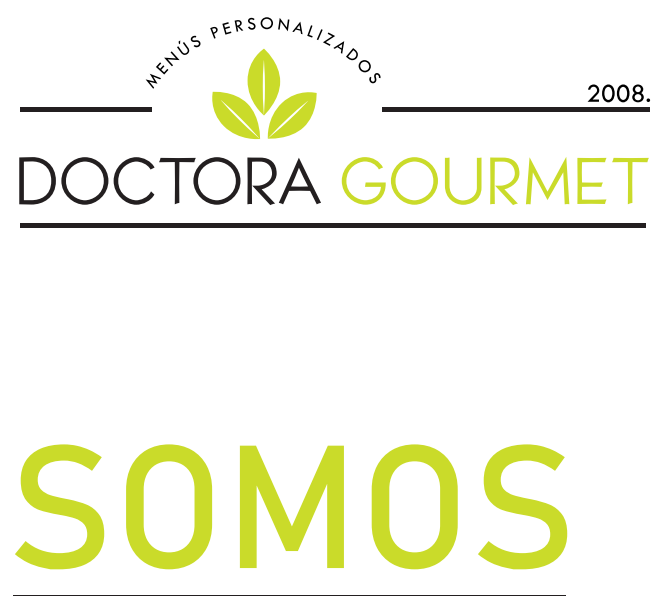

\section{ALIMENTACIÓN FUNCIONAL}

MENÚS 100\% PERSONALIZADOS

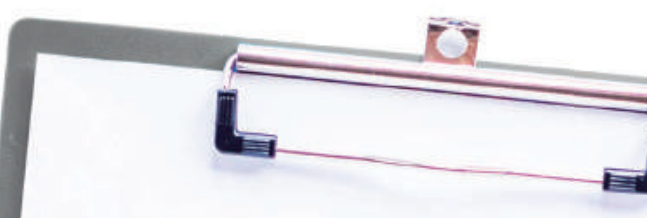

DIABÉTES HIPERTENSIÓN OBESIDAD

METABOLISMO COLESTEROL COLONIRRITABLE
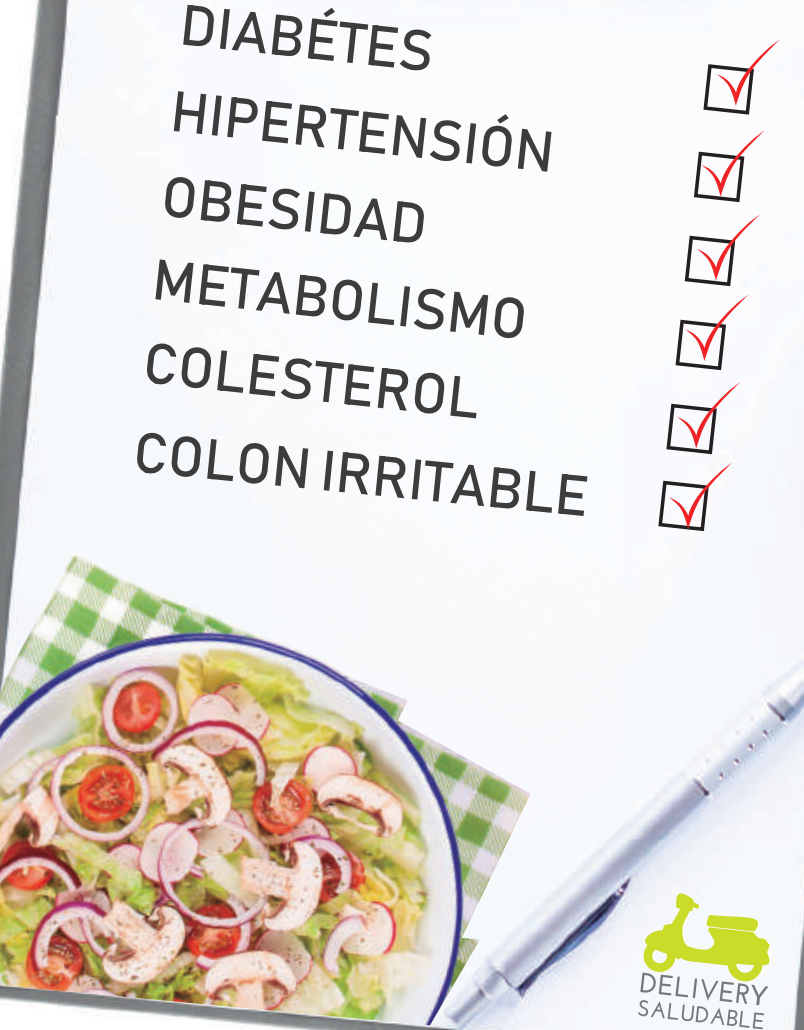